\title{
Design and characterization of a linear quadrupole ion trap for high-resolution Coulomb-crystal time-of-flight mass spectrometry
}

\author{
Daniel Rösch, Hong Gao, Ardita Kilaj and Stefan Willitsch*
}

\author{
${ }^{*}$ Correspondence: \\ stefan.willitsch@unibas.ch \\ Department of Chemistry, \\ University of Basel, \\ Klingelbergstrasse 80, 4056 Basel, \\ Switzerland
}

\begin{abstract}
We present and discuss in detail the design and characterization of a new linear quadrupole ion trap with additional ion ejection and acceleration electrodes that is coupled to a time-of-flight mass spectrometer. Mass spectra of Coulomb crystals consisting of $\mathrm{Ca}^{+}, \mathrm{CaO}^{+}$and $\mathrm{CaOH}^{+}$ions were recorded with a post-ejectionacceleration scheme yielding a mass resolution of $m / \Delta m \approx 700$. The second order rate constant for the reaction $\mathrm{Ca}^{+}+\mathrm{N}_{2} \mathrm{O} \rightarrow \mathrm{CaO}^{+}+\mathrm{N}_{2}$ was measured to test the usability of this apparatus for ion-molecule reaction studies. The rate constant was found to be $5.49(32) \cdot 10^{-11} \mathrm{~cm}^{3} \mathrm{~s}^{-1}$ which is compared with previous literature values. Owing to the high mass resolution achieved, the present instrument is an ideal tool for the study of the products of complex chemical reactions involving Coulomb crystals.

Keywords: Ion trap, Time-of-flight mass spectrometry, Ion-molecule reactions, Coulomb crystals
\end{abstract}

\section{Introduction}

Linear quadrupole radiofrequency (RF) ion traps (LQT) are widely used instruments for the trapping and cooling of atomic and molecular ions. Cold, trapped ions are of interest for a variety of topics, ranging from precision spectroscopy [1] and quantum information [2] to the dynamics of ion-molecule reactions [3-5]. Laser- and sympathetically-cooled ions in traps form ordered structures termed Coulomb crystals [6]. Rate constants for molecules reacting with the laser cooled or sympathetically cooled ions can be determined by observing changes in the crystal structure [6-10]. The mass-to-charge ratio of the ionic reaction products can be determined by resonant-excitation mass spectrometry (REMS) $[8,11,12]$. REMS works best on small Coulomb crystals consisting of one single atomic or molecular ion that is sympathetically cooled by one laser cooled ion enabling the distinction of isotopes that differ by only one atomic mass unit $(u)[12,13]$. However, for larger mixed-species Coulomb crystals the REMS spectra become complex due to Coulomb coupling [14] and an unambiguous mass assignment is not always possible $[15,16]$.

The development of molecular-beam machines coupled to LQTs enables the study of highly controlled ion-molecule reaction dynamics by allowing to select specific velocities,

() 2016 Rösch et al. licensee Springer on behalf of EPJ. This is an Open Access article distributed under the terms of the Creative Commons Attribution License (http://creativecommons.org/licenses/by/4.0), which permits unrestricted use, distribution, and reproduction in any medium, provided the original work is properly cited. 
quantum states and conformations of the neutral molecules [3, 10, 15-17]. Ion-molecule reactions with large organic molecules can have several channels that lead to a multitude of product ion species. To improve the resolution of the mass analysis and quantify the amount of trapped ions, LQTs have been coupled to time-of-flight (TOF) mass spectrometers (MS) in several recent experiments.

Schowalter et al. developed a LQT where the extraction field is applied directly to the trap electrodes [18]. This mass spectrometer featured a resolution of $m / \Delta m \approx 50$ and an improved version achieved $m / \Delta m \approx 500$ for trapped laser-cooled ions [19]. The trapping RF potential is switched off fast, just before the ions are ejected into the flight tube, requiring custom electronics to combine quickly switchable, high-amplitude RF and DC fields [20].

Deb et al. developed a "digital" ion trap from which Coulomb crystals of laser cooled and sympathetically cooled ions are ejected between square wave pulses. With this method, they were able to reach a mass resolution of $m / \Delta m \approx 90$ [21]. Meyer et al. built a trap in which the RF with a standard cosine waveform is damped prior to the ejection of the ions that achieves a mass resolution of $m / \Delta m \approx 100$ and compared its performance to the digital ion trap [22]. Ni et al. reported a six-rod ion trap from which ions are ejected radially onto an MCP detector for photodissociation studies of $\mathrm{HfF}^{+}$[23]. Jyothi et al. developed a wire-electrode trap where the ions are ejected while the RF remains on. They observed a strong dependence of the ion flight time on the RF phase angle at the time of ejection leading to complex mass spectra which are difficult to interpret if more than one ion species are trapped and ejected [24]. In all of these experiments, the ions are ejected radially from the trap. Seck et al. coupled a LQT to a TOF-MS in which the ions are accelerated along the trap axis into the TOF-MS by applying a potential gradient using the endcaps of the ion trap [25]. The advantage of this setup is the separation of RF and highvoltage electrodes but the mass resolution for heavy ions in multi-component Coulomb crystals seems limited.

In a recent experiment, we studied the conformer specific reaction rate constants of 3 -aminophenol with $\mathrm{Ca}+$ Coulomb crystals $[15,16]$. REMS was used to analyze the product ions, but an unambiguous assignment was not possible because two possible products differ by only $1 \mathrm{u}$. Therefore, we recently developed a new experimental setup featuring a new LQT that is coupled to a TOF-MS. The ions are ejected radially using additional electrodes inside the trap [26]. The trapping RF is turned off prior to the ion ejection. Additional electrodes are installed below the trap to accelerate and focus the ions onto an MCP detector. With this post-ejection-acceleration scheme, a high mass resolution of $m / \Delta m \approx 700$ can be achieved over a narrow mass range defined by the time delay between ejection and acceleration. By varying this time delay, the entire mass range of interest can be probed with high resolution. Our new LQT-TOF-MS features a design with separate RF and ejection DC electrodes, thereby enabling operation with commercially available RF and DC electronics.

\section{Experimental setup}

Our new ion trap is a LQT with additional electrodes embedded for ion ejection into a time-of-flight mass spectrometer. Figure 1 shows a schematic of the ion trap and vacuum apparatus. The trap electrodes consist of stainless steel rods that are segmented into three parts with two outer electrodes (endcaps) of $20 \mathrm{~mm}$ length and a center segment of 


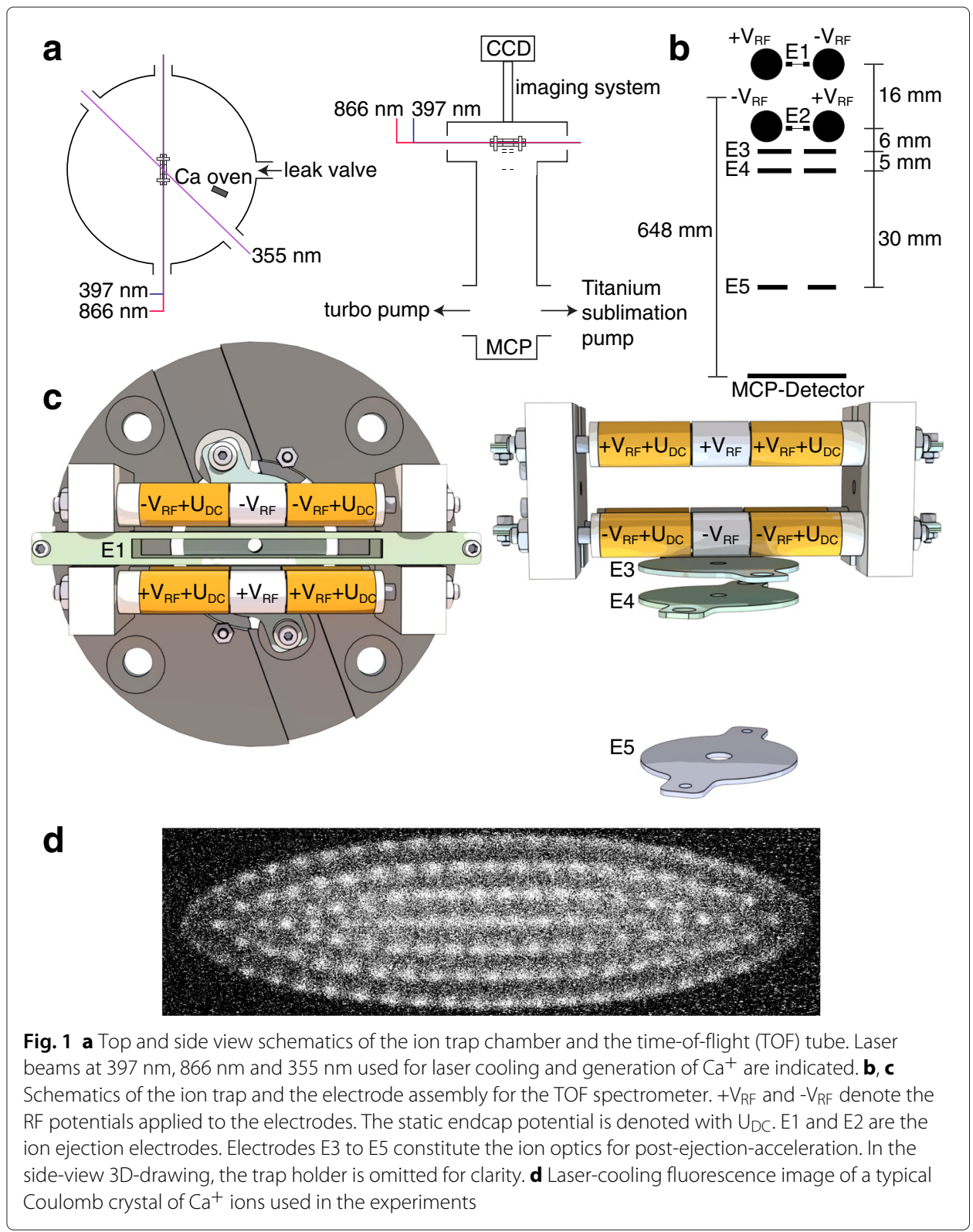

$2 z_{0}=10 \mathrm{~mm}$ length. The electrode diameter is $8 \mathrm{~mm}$ and the diagonal distance between electrode surfaces is $2 r_{0}=14.63 \mathrm{~mm}$. Between the top and bottom pair of cylindrical electrodes two additional rectangular electrodes are mounted. These electrodes consist of two $0.5 \mathrm{~mm}$ thick stainless steel parts with a $50 \mathrm{~mm}$ long and $3 \mathrm{~mm}$ wide gap. A nickel mesh (Precision Eforming) with 90 \% ion transmission was clamped in between the two pieces and the frame was spot welded together to fix the mesh in place. All electrodes are held in place by two MACOR ${ }^{\circ}$ holders at both ends of the trap. Beneath the ion trap, three circular, $1 \mathrm{~mm}$ thick stainless steel electrodes are suspended from the trap mount to form the ion optics for the TOF-MS. The two top plates are separated vertically by 5 $\mathrm{mm}$ having each a $3 \mathrm{~mm}$ wide hole in the center. The distance from the trap center to the first plate is $14 \mathrm{~mm}$. The third electrode is $30 \mathrm{~mm}$ below the second plate and has a $5 \mathrm{~mm}$ 
diameter hole in the center. The TOF-trap is mounted on a breadboard inside a spherical octagon vacuum chamber (Kimball Physics). A micro-channel-plate (MCP) detector (Photonis APD 2 APTOF) with an active diameter of $40 \mathrm{~mm}$ is mounted $648 \mathrm{~mm}$ below the trap center. The trap chamber and the flight tube are differentially pumped by $65 \mathrm{l} / \mathrm{s}$ and $550 \mathrm{l} / \mathrm{s}$ turbomolecular pumps connected to a $16500 \mathrm{l} / \mathrm{h}$ foreline pump. To efficiently reduce the hydrogen partial pressure, a titanium sublimation pump is connected to the vacuum system. The base pressure in this setup is $<5 \cdot 10^{-10} \mathrm{mbar}$.

${ }^{40} \mathrm{Ca}^{+}$ions are generated by non-resonant multi photon ionisation of an atomic beam of $\mathrm{Ca}$ by the third harmonic $(355 \mathrm{~nm}$ ) of a Nd:YAG laser. The Ca beam is generated by a resistively heated $\mathrm{Ca}$ oven (Alvatec). The ${ }^{40} \mathrm{Ca}^{+}$ions are laser cooled by two laser beams at $397 \mathrm{~nm}$ and $866 \mathrm{~nm}$ propagating along the trap axis [6]. The 397 and $866 \mathrm{~nm}$ light is generated by two external cavity-enhanced diode lasers that are frequency stabilized using a wavemeter (HighFinesse WSU 30) and a computer controlled feedback loop. A leak valve allows for the controlled admission of gas to the trap chamber. Coulomb crystals of ${ }^{40} \mathrm{Ca}^{+}$are imaged using an electron multiplier charge coupled device (EMCCD) camera (Andor Luca) mounted on a microscope with a magnification of $\sim 12.6$. The trap is driven by a commercially available radio frequency generator (Stahl-Electronics HF-DR 3.5-900 FL) which can be switched off within one RF cycle. The trap is operated on resonance at $\Omega=2 \pi \times 3.275 \mathrm{MHz}$ and peak-to-peak RF amplitude $V_{\mathrm{RF}, \mathrm{pp}}=800 \mathrm{~V}$. The RF voltage is applied to all trap segments with opposite phases on adjacent rods and is mixed with typically $5 \mathrm{~V}$ DC voltage on the endcap segments. Ion extraction and acceleration potentials are applied by fast high-voltage switches (Behlke HTS 61-01-HB-C) with a 10$90 \%$ rise time of $\sim 70 \mathrm{~ns}$. The MCP signal is amplified by a homebuilt $26 \mathrm{~dB}$ amplifier and recorded on a $600 \mathrm{MHz}$ oscilloscope (LeCroy Wavesurfer 64 MXs). The high voltage switches and the data acquisition are triggered by a pulse generator (Quantum Composer 9520 series).

Our LQT-TOF-MS setup can be operated in two different modes. In low resolution mode, a high voltage pulse is applied only to the top extraction mesh. In high resolution mode, additional focusing potentials are applied to the ion optics below the trap after the ions have left the trap.

\section{Experimental results and discussion}

The electrostatic trap potential was modeled numerically in Simion 8.1 [27]. A three dimensional fourth-order polynomial was fitted to the numerical potential inside a $4 \times 4 \times 4 \mathrm{~mm}$ cube around the trap center. The fitted potential was used as input for generating a force field in molecular dynamics (MD) simulations using the Protomol software $[28,29]$. Figure 2 shows the stability diagram $[30,31]$ for a single ${ }^{40} \mathrm{Ca}^{+}$ion in the trap as obtained from MD simulations for an ion at room temperature without laser cooling. If for a specific set of Mathieu a and q parameters [30] the ion was found in a volume of $1 \mathrm{~mm}^{3}$ around the trap center after $1000 \mathrm{RF}$ cycles, it was considered to be stably trapped.

Figure 3 shows a 2D cut through the total time-averaged trapping potential (pseudopotential [30] and static potentials) at the trap center and 1D cuts along the 3 principal axes. Due to the extraction meshes at the top and bottom of the trap, the potential gets distorted such that it becomes weaker along the $y$ axis than along the $x$ axis. The trap depth (defined as the difference between the potential minimum at the trap center and 


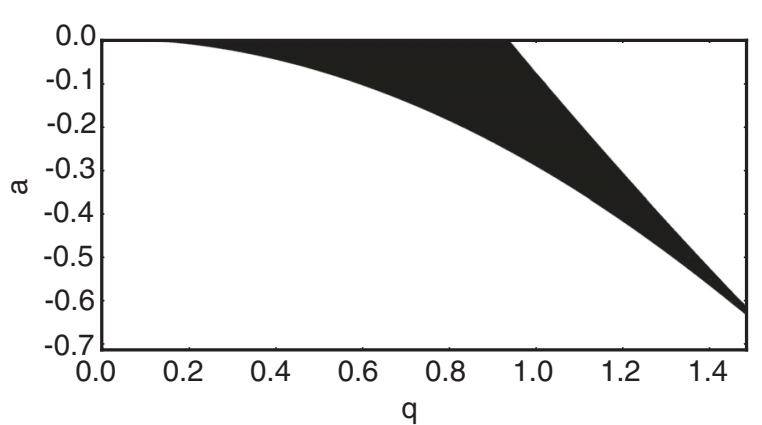

Fig. 2 Stability diagram generated from MD simulations of a single $\mathrm{Ca}^{+}$ion at room temperature inside the ion trap. Trap frequency $\Omega=2 \pi \times 3.275 \mathrm{MHz}$

the nearest maximum in the $1 \mathrm{D}$ potential cuts) is $11.29 \mathrm{eV}$ in the $\mathrm{x}$ dimension and $2.43 \mathrm{eV}$ in the y dimension at $\Omega=2 \pi \times 3.275 \mathrm{MHz}$ and $V_{\mathrm{RF}, \mathrm{pp}}=800 \mathrm{~V}$.

This asymmetry in the potential leads to asymmetric Coulomb crystals. While ions lighter than $\mathrm{Ca}^{+}$gather at the trap center, heavier ions form surrounding shells. The latter are more susceptible to the asymmetric trapping potential which results in their distribution being stretched along the $y$ axis. The shape of the crystals can be controlled by applying a DC potential to the top and bottom mesh. Figure 4a shows the results of MD

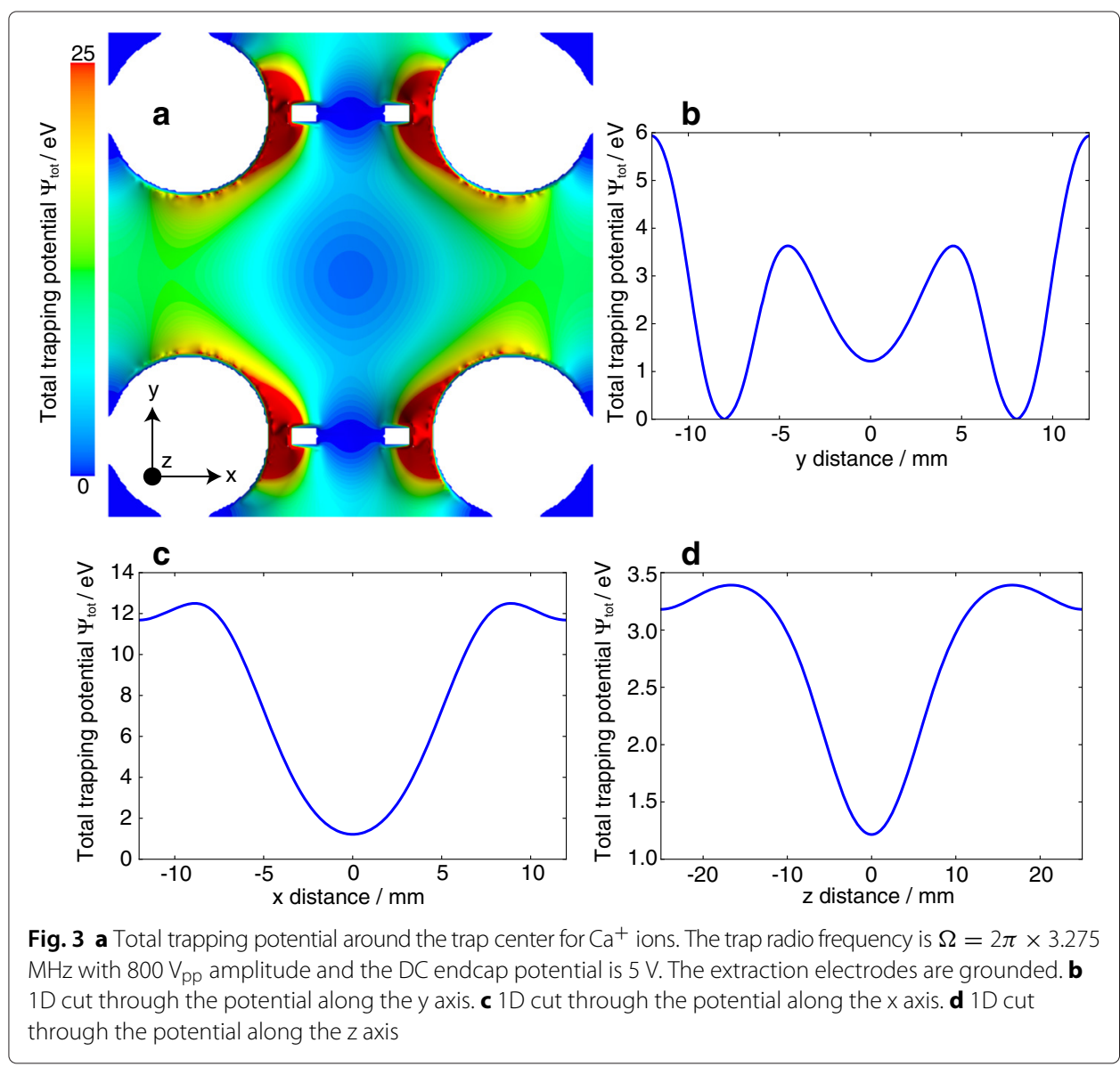


a
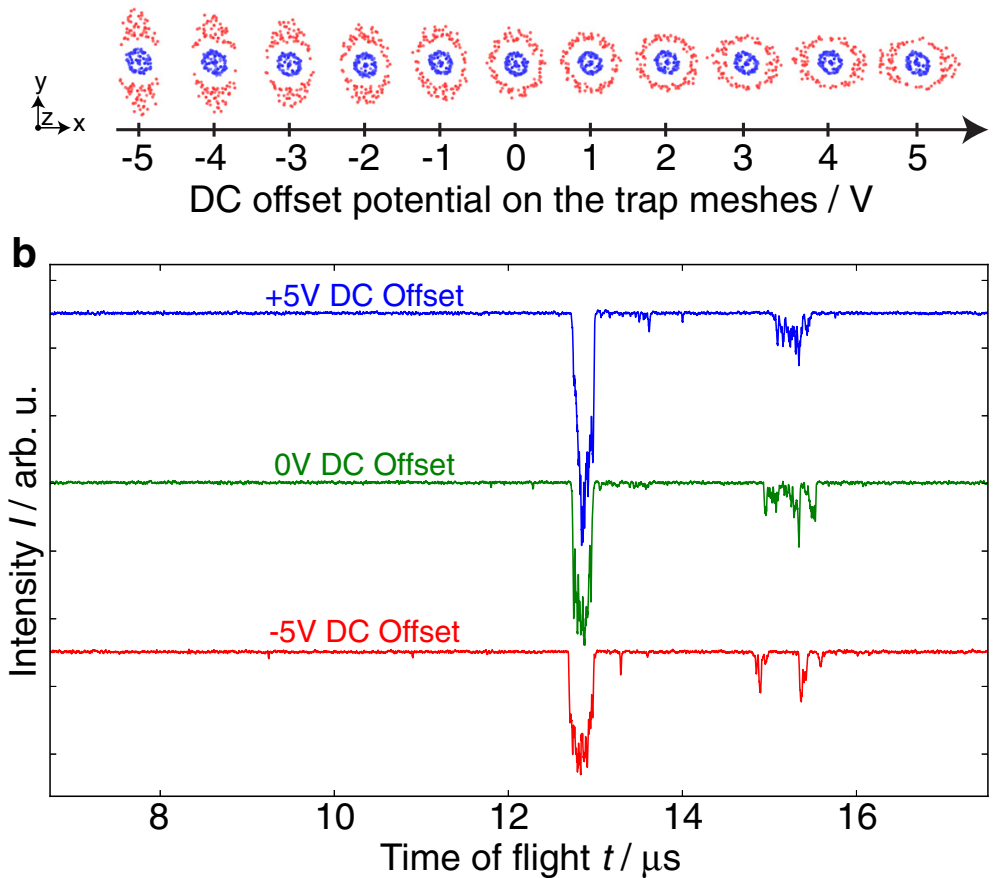

Fig. 4 a Molecular dynamics simulations of $100 \mathrm{Ca}^{+}$(blue) and 100 ions of mass $80 \mathrm{u}$ (red) at different DC offset potentials applied to the extraction meshes. $\mathbf{b}$ Time-of-flight traces of Coulomb crystals consisting of $\mathrm{Ca}^{+}, \mathrm{CaO}^{+}$and $\mathrm{CaOH}^{+}$ions at different DC offset potentials applied to the extraction meshes prior to the ion extraction. Each trace is the average of 5 measurements

simulations of Coulomb crystals consisting of $100 \mathrm{Ca}^{+}$ions and 100 ions with a mass of $80 \mathrm{u}$ at different DC offsets applied to the extraction meshes.

Figure $4 \mathrm{~b}$ shows measured low-resolution time-of-flight (TOF) spectra of mixed species Coulomb crystals consisting of $\mathrm{Ca}^{+}, \mathrm{CaO}^{+}$and $\mathrm{CaOH}^{+}$ions at different $\mathrm{DC}$ offsets applied to the extraction meshes. $\mathrm{CaO}^{+}$ions were created by leaking $\mathrm{N}_{2} \mathrm{O}$ into the chamber to react with $\mathrm{Ca}^{+}$in the reaction $\mathrm{Ca}^{+}+\mathrm{N}_{2} \mathrm{O} \rightarrow \mathrm{CaO}^{+}+\mathrm{N}_{2}$ [32-34]. $\mathrm{CaOH}^{+}$was formed from $\mathrm{Ca}^{+}$reacting with water which was introduced into the vacuum chamber as a contamination of the $\mathrm{N}_{2} \mathrm{O}$ gas. The TOF signal at $12.9 \mu$ s arises from the ${ }^{40} \mathrm{Ca}^{+}$ions. The signal around $15.5 \mu \mathrm{s}$ is caused by $\mathrm{CaO}^{+}$and $\mathrm{CaOH}^{+}$ions. Changing the $\mathrm{DC}$ offset applied to the extraction meshes strongly influences the signal shape, especially for the heavy ions, and confirms the asymmetric crystal shapes predicted by the MD simulations (the terms "heavy" and "light" ions are used with respect to the mass of ${ }^{40} \mathrm{Ca}^{+}$). At $-5 \mathrm{~V}$ DC offset the heavy ions form two separate distributions above and below the $\mathrm{Ca}^{+}$crystal core. These two ion packets arrive at the MCP detector at different times and give rise to two separate TOF signals. At positive offsets the crystal is squeezed along y such that heavy ions preferentially lie in the horizontal plane and reach the detector at the same time. Thus, they produce a single peak in the $+5 \mathrm{~V}$ TOF trace. For light ions, the trap asymmetry does not significantly influence the crystal shape. This is confirmed experimentally for the $\mathrm{Ca}^{+}$ions for which the peak width is not so strongly influenced by the DC offset. In conclusion, the trap asymmetry can be corrected by applying an appropriate DC offset to the extraction meshes. In the case of the artifical crystal consisting of 100 
$\mathrm{Ca}^{+}+100$ ions of mass $80 \mathrm{u}$, an offset of $1 \mathrm{~V}$ produces rotationally symmetric crystals according to the MD simulations.

The TOF spectra in Fig. 4 exhibit a low resolution $m / \Delta m \approx 30$ for the $\mathrm{Ca}^{+}$signal. After turning off the trapping RF and waiting for $0.1 \mu \mathrm{s}$, the ions were ejected from the trap by applying a $1.0 \mu$ s long $4000 \mathrm{~V}$ pulse to the top mesh. Figure 5 shows the extraction potential inside the trap while $4000 \mathrm{~V}$ are applied to the top mesh. The extraction mesh is partially shielded by the trap electrodes held at ground potential which results in a weakening of the extraction field at the position of the ions. Moreover, the field is inhomogeneous. This leads to long flight times $t$ and broad peaks. Ideally, the TOF extraction region should resemble a Wiley-McLaren setup with a two-stage acceleration region for obtaining a high resolution $[18,21,35]$. With the trap electrodes at ground potential during ion extraction, a Wiley-McLaren-type extraction region is not feasible. If high voltages are applied to the bottom extraction mesh the ions are lost from the trap along the $\mathrm{x}$ axis. For low potentials the ions are strongly defocused and do not reach the detector either.

To improve the resolution of the spectrometer, we employ a post-ejection-acceleration scheme in which the ions experience an additional accelerating potential after they have left the trap. Figure $6 c$ shows the pulse sequence used to accelerate the ions. The RF voltage is damped within a few cycles (see Fig. 6b) and after $0.1 \mu \mathrm{s}$, a voltage of $4000 \mathrm{~V}$ is applied to the top extraction mesh. Simulations showed that the residual damped RF present during extraction does not strongly affect the TOF resolution. After a delay $\Delta t$, a voltage of $4000 \mathrm{~V}$ is pulsed to the top accelerator plate (E3) below the trap and a potential of $3200 \mathrm{~V}$ is applied to the middle accelerator plate (E4) while the third accelerator plate (E5) is held at ground potential (Fig. 6a). The potentials applied to E3 and E4 are generated by a single high voltage pulser with a home made voltage divider. The optimal acceleration delay $\Delta t$ is highly mass dependent and only one species at a time can be efficiently focused. Accordingly, the delay has to be adjusted in order to optimize the resolution for a certain ion species.

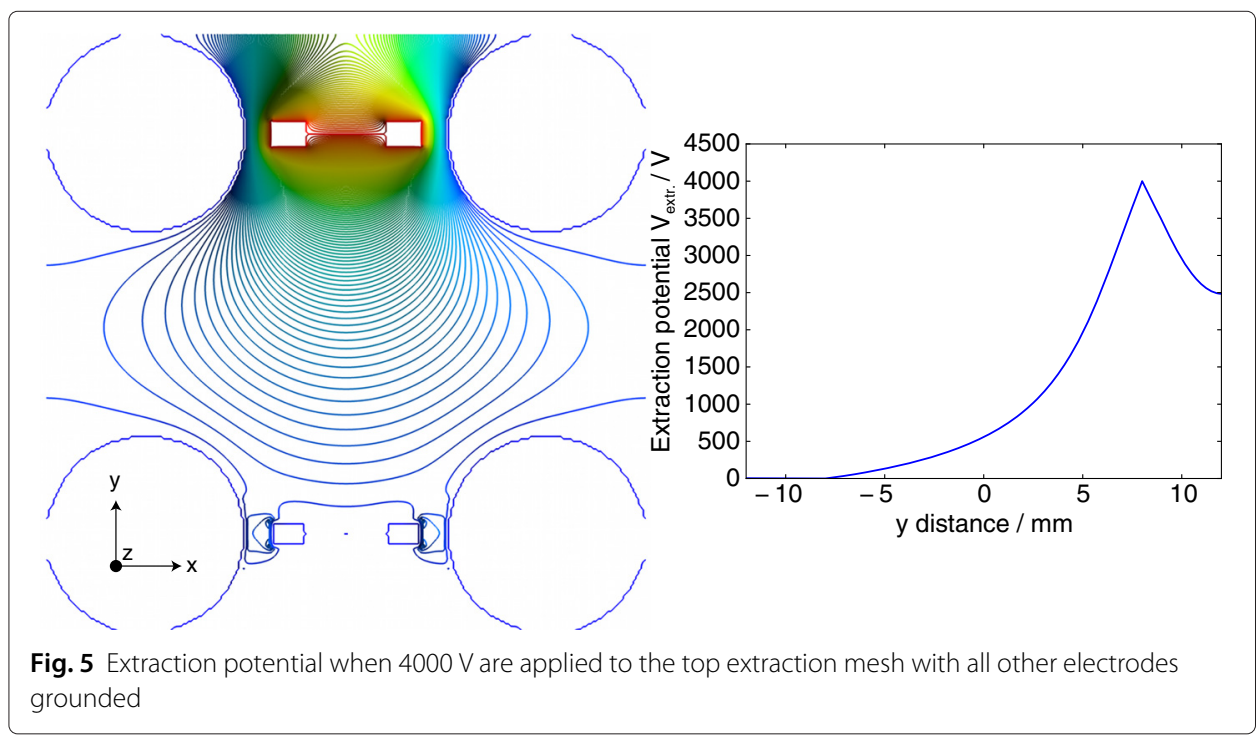




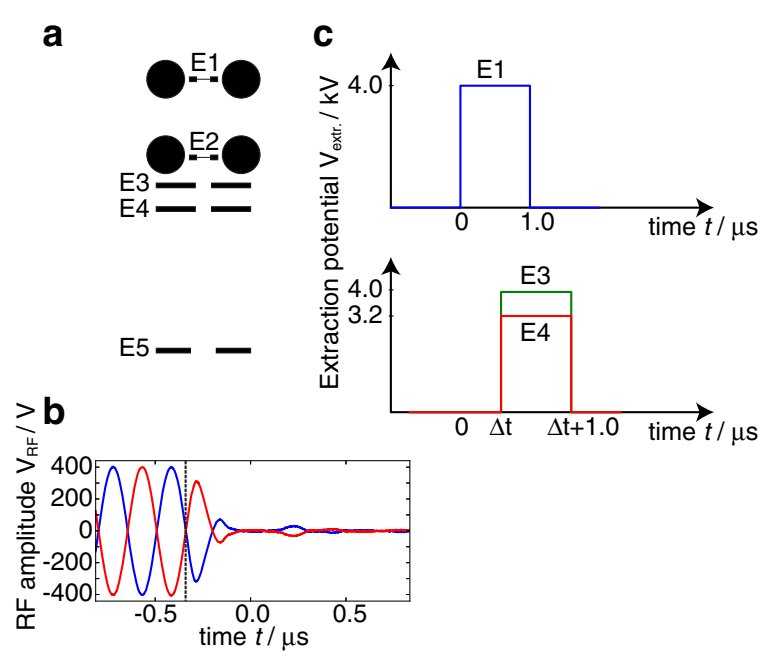

Fig. 6 a Schematic representation of the ion trap with the extraction and acceleration electrodes labeled E1-E5. b Oscilloscope traces of both radio frequency phases at the time when the RF generator is switched off. The dashed line indicates the switch-off time. $\mathbf{c}$ High voltage pulse sequence to eject ions from the trap and accelerate them. The optimal value for $\Delta \mathrm{t}$ is dependent on the ion mass-to-charge ratio. See text for details

Figure 7a shows the results of a scan of the acceleration delay for mixed Coulomb crystals consisting of $\mathrm{Ca}^{+}, \mathrm{CaO}^{+}$and $\mathrm{CaOH}^{+}$ions. The experiment was conducted in the following way: $\mathrm{A} \mathrm{Ca}^{+}$Coulomb crystal was loaded and cleaned from heavy impurity ions by lowering the RF amplitude allowing all ions with mass $>40 \mathrm{u}$ to leave the trap. After ramping the RF amplitude back up to $V_{\mathrm{RF}, \mathrm{pp}}=800 \mathrm{~V}, \mathrm{~N}_{2} \mathrm{O}$ was leaked into the chamber at a partial pressure of $\sim 8 \cdot 10^{-9}$ mbar to form $\mathrm{CaO}^{+}$ions. The $\mathrm{CaO}^{+}$ions were sympathetically cooled into the Coulomb crystal and the reaction was monitored by observing

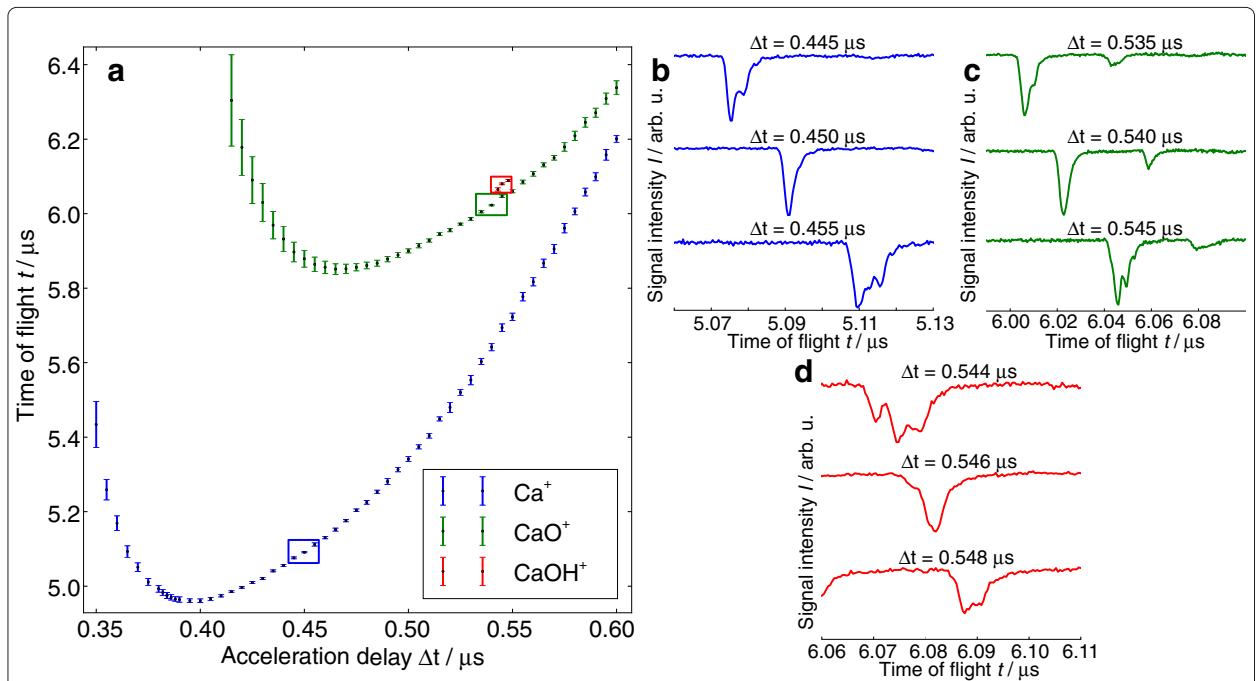

Fig. 7 a Plot of the post-ejection-acceleration time delay versus the ion time-of-flight for 3 different ion species. Each data point corresponds to the peak center of an average over 5 TOF traces. The error bars give the FWHM of the peaks. b Averaged TOF traces at acceleration delays that produce the sharpest $\mathrm{Ca}^{+}$signal. Each trace is an average over 5 experiments. c Averaged TOF traces at acceleration delays that produce the sharpest $\mathrm{CaO}^{+}$signal. Each trace is an average over 5 experiments. $\mathbf{d}$ Averaged TOF traces at acceleration delays that produce the sharpest $\mathrm{CaOH}^{+}$signal. Each trace is an average over 5 experiments 
the disappearance of $\mathrm{Ca}^{+}$ions. The reaction was stopped, by closing the leak valve, when about half of the $\mathrm{Ca}^{+}$crystal had reacted away. The Coulomb crystal was then ejected into the TOF spectrometer and accelerated after a specific post-ejection-acceleration delay. Each data point in Fig. 7 gives the peak center of an average over 5 TOF traces and the error bars correspond to the full width at half maximum (FWHM) of the averaged signal indicating the TOF resolution. If the post-ejection accelerator is switched on too early, the ions are repelled upwards and do not reach the detector. This is the case for the $\mathrm{CaO}^{+}$ ions at accelerator delays $<0.415 \mu \mathrm{s}$. For all measured ions, we observed that the flight time is a convex function of the acceleration delay with a global minimum. The optimal delay that produces the strongest and narrowest signal is usually found slightly after the minimum of the curve. To analyze the TOF spectra and to extract the mass-to-charge $(m / q)$ ratio of the measured ions, one needs to find the acceleration delay with the best focusing condition for each ion species. We assume that the optimal ion position inside the accelerator, at the time when it is switched on, is the same for all ionic species independent of their $m / q$ ratio. This means we can calculate the mass of an unknown species from its flight time at the optimal accelerator delay compared to the TOF of another ionic species which serves as a reference at its own optimal accelerator delay.

To test this assumption, we measured the mass of the $\mathrm{CaO}^{+}$ions using ${ }^{40} \mathrm{Ca}^{+}$as a reference. Figure $7 \mathrm{~b}$ and $\mathrm{c}$ show the averaged TOF traces around the optimal acceleration delay for the $\mathrm{Ca}^{+}(\mathrm{B})$ and $\mathrm{CaO}^{+}(\mathrm{C})$ ions. Acceleration delays of $0.45 \mu \mathrm{s}$ for $\mathrm{Ca}^{+}$and 0.54 $\mu \mathrm{s}$ for $\mathrm{CaO}^{+}$give the narrowest signals. The resolution $m / \Delta m$ is defined as $t / 2 \Delta t$ with $\Delta t$ being the FWHM of the peak. At the ideal acceleration delay, the resolution is 878 for the $\mathrm{Ca}^{+}$signal and 669 for $\mathrm{CaO}^{+}$. The broadening of the signal at non-optimal delays is caused by a temporal jitter between the extraction mesh pulser and the accelerator pulser. Its effect is amplified if the ions are not at the correct position inside the accelerator at the time when the accelerator pulser is switched on.

For these experiments, Coulomb crystals with a constant size of approximately 600 ions were used. Without the post-ejection acceleration, we observed a decrease of the mass resolution by a factor $\approx 2$ when increasing the size of the crystal from $\approx 100$ to $\approx 600$ ions. By comparison, when using post-ejection acceleration, the dependence of the mass resolution on the crystal size is much less pronounced and only accounts for a variation of $\approx 15 \%$ in the size interval sampled in Fig. 8b below. The error bars in Fig. 7a indicate the full-width-at-half-maximum (FWHM) of the peaks in the TOF spectrum illustrating the variation of the mass resolution for different masses at different acceleration delays.

Using the relation $t_{\mathrm{CaO}^{+}} / t_{\mathrm{Ca}^{+}}=\sqrt{m_{\mathrm{CaO}^{+}} / m_{\mathrm{Ca}^{+}}}$with $m_{\mathrm{Ca}^{+}}=39.963 \mathrm{u}$ as the mass of ${ }^{40} \mathrm{Ca}$, we calculate $m_{\mathrm{CaO}^{+}}=55.93(11) \mathrm{u}$ for the $\mathrm{CaO}^{+}$signal at the $0.54 \mu$ s acceleration delay. This mass agrees well with the exact mass of $\mathrm{CaO}(55.958 \mathrm{u})$. In Fig. 7c a weak signal at later flight times is observed. This signal is caused by $\mathrm{CaOH}^{+}$which is formed from the reaction of $\mathrm{Ca}^{+}$ions with water impurities in the gas. To improve the signal to noise ratio and prove that this signal is from $\mathrm{CaOH}^{+}$, we reduced the $\mathrm{N}_{2} \mathrm{O}$ pressure to $\sim 2 \cdot 10^{-9}$ mbar and let the crystals react for a longer time which resulted in the formation of a larger amount of $\mathrm{CaOH}^{+}$. We scanned the acceleration delay and found the strongest and sharpest signal for a delay of $0.546 \mu \mathrm{s}$. The mass of this signal is 57.04(10) $\mathrm{u}$ which agrees well with the exact mass of $\mathrm{CaOH}(56.965 \mathrm{u})$. Table 1 gives an overview over all results from the post-ejection-acceleration TOF experiments. 


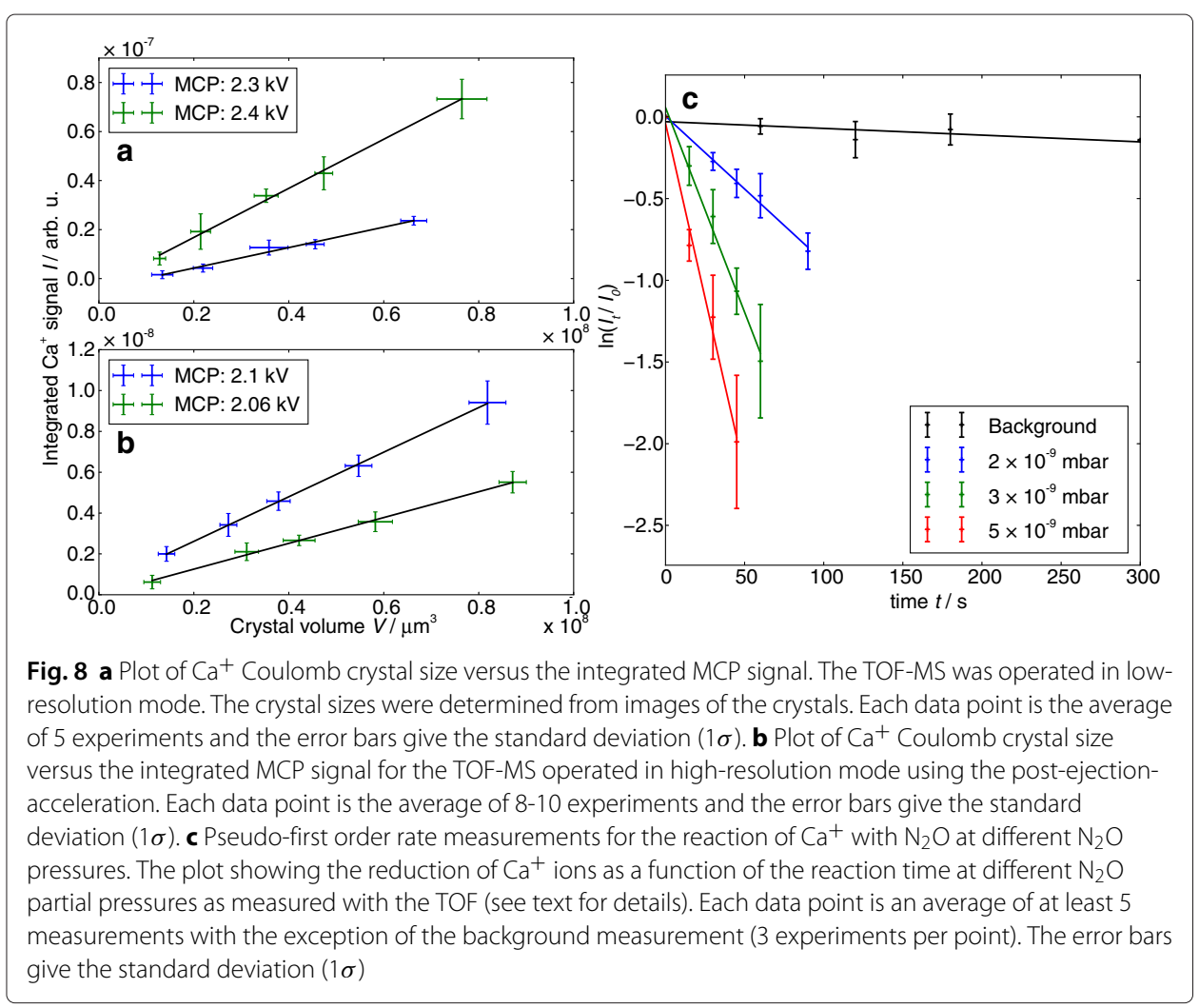

The general procedure to measure the mass of an unknown ion $\mathrm{x}$ is to first measure a low resolution TOF spectrum and then estimate the best post-ejection-acceleration delay from the relation $t_{x} / t_{C a^{+}}=\Delta t_{x} / \Delta t_{C a^{+}}$. One then measures TOF spectra using postejection-acceleration and optimizes $\Delta t$ to achieve the best-resolved signal. The mass can be calculated from the ion TOF at the relevant optimized accelerator time delay using $t_{x} / t_{\mathrm{Ca}^{+}}=\sqrt{m_{x} / m_{C a^{+}}}$.

Our ion trap is designed so that the ions can also be ejected while the RF is applied. Similar to Jyothi et al. [24], we see a strong RF phase angle dependence on the ion ejection efficiency and their flight time. This resulted in very complicated spectra. In order to unambiguously assign the masses of all ions in a multi component crystal, we had to average the TOF spectra over all RF phases which reduced the resolution by an order of magnitude and the distinction between $\mathrm{CaO}^{+}$and $\mathrm{CaOH}^{+}$was not possible anymore. For high resolution TOF spectra that are easy to analyze, it is necessary to switch the RF off before ejecting the ions. An important application of this setup lies in the determination of the rates of ion-molecule reactions which necessitates quantitatively correct counts of ions. Previously, images of Coulomb crystals were used to calculate the crystal volume

Table 1 TOF post-ejection-acceleration results for $\mathrm{Ca}^{+}, \mathrm{CaO}^{+}$and $\mathrm{CaOH}^{+}$ions

\begin{tabular}{|c|c|c|c|c|c|c|}
\hline Ion species & $\begin{array}{l}\text { Optimal acceleration } \\
\text { delay } \Delta t / \mu \mathrm{s}\end{array}$ & $\begin{array}{l}\text { Time of flight } \\
t / \mu s\end{array}$ & $\mathrm{FWHM} / \mathrm{ns}$ & $\begin{array}{l}\text { mass resolution } \\
m / \Delta m\end{array}$ & Expt. mass $m / u$ & $\begin{array}{l}\text { Exact mass } \\
m / u\end{array}$ \\
\hline $\mathrm{Ca}^{+}$ & 0.450 & 5.091 & 2.9 & 878 & - & 39.963 \\
\hline $\mathrm{CaO}^{+}$ & 0.540 & 6.023 & 4.5 & 669 & $55.93(11)$ & 55.958 \\
\hline $\mathrm{CaOH}^{+}$ & 0.546 & 6.082 & 4.2 & 724 & $57.04(10)$ & 56.965 \\
\hline
\end{tabular}


assuming that the crystal is rotationally symmetric in a standard LQT. From the change of the crystal volume over time, reaction rates could be extracted [9, 15-17].

This method is difficult to use when several ionic species are involved in a reaction and rates have to be determined for all channels. Only the $\mathrm{Ca}^{+}$ions are detected by fluorescence imaging and changes in the crystal shape might be very subtle so that even with the help of MD simulations, it might be difficult or impossible to determine which ions and how many have reacted. To improve this situation, we want to use the TOF mass spectrometer to monitor the reaction progress by ejecting the Coulomb crystal after a certain time. The integral over the MS signal of a specific ion is proportional to the number of trapped ions at the time of ejection.

To test whether we can use our spectrometer to quantify the amount of ions in the trap, we measured TOF spectra of $\mathrm{Ca}^{+}$Coulomb crystals of different sizes and integrated over the $\mathrm{Ca}^{+}$signal. The crystal sizes were deduced from the crystal images. Figure 8a shows the results of this experiment. The crystal size linearly correlates with the integrated MCP signal for all tested crystal sizes. These experiments were done with the TOF-MS in low resolution mode by only using the top extraction mesh. We repeated this experiment with the post-ejection-acceleration switched on and observed the same linear relationship between the crystal size and the integrated detector signal (Fig. 8b).

To show that we can measure rate constants with this setup, we observed the reaction of $\mathrm{Ca}^{+}$ions with $\mathrm{N}_{2} \mathrm{O}$ and determined the rate constant. Coulomb crystals of $\mathrm{Ca}^{+}$ions were prepared and $\mathrm{N}_{2} \mathrm{O}$ was leaked into the vacuum chamber at varying partial pressures. After a certain time, the Coulomb crystal was ejected into the TOF-MS which was operated in low resolution mode. This reaction can be treated with pseudo-first order kinetics since the $\mathrm{N}_{2} \mathrm{O}$ is available in large excess. For pseudo-first order kinetics, the integrated rate law $\ln \left(I_{t} / I_{0}\right)=-k N_{\mathrm{N}_{2} \mathrm{O}} t$ holds, where $I_{t}$ and $I_{0}$ are the integrated TOF signals of the reactants at time $t$ and $t=0$, respectively, $k$ is the bimolecular rate constant and $N_{\mathrm{N}_{2} \mathrm{O}}$ is the $\mathrm{N}_{2} \mathrm{O}$ density. To determine $I_{t}$ we integrate the $\mathrm{Ca}^{+}$signal of the TOF trace, for $I_{0}$ we use the crystal size versus MCP calibration curve in Fig. 8a and apply it to the image of the Coulomb crystal before the reaction. Figure $8 \mathrm{c}$ shows $\ln \left(I_{t} / I_{0}\right)$ versus $\mathrm{t}$ for three different $\mathrm{N}_{2} \mathrm{O}$ partial pressures in the range from $2 \cdot 10^{-9}$ to $5 \cdot 10^{-9}$ mbar and a background reaction in which no $\mathrm{N}_{2} \mathrm{O}$ was admitted into the chamber. The data clearly shows a linear relationship and pseudo-first order rate constants can be obtained from linear fits.

The second order rate constant calculated from this data is $k=4.2(10) \cdot 10^{-10} \mathrm{~cm}^{3} \mathrm{~s}^{-1}$ which is a factor of 3 to 10 larger than other values reported in the literature for this reaction at room temperature [32-34]. The error quoted for our value of $k$ represents the uncertainty of the fit to the experimental data.

To understand this discrepancy, one has to consider that the ions are laser cooled during the reaction. Thus, part of the $\mathrm{Ca}^{+}$ions are in electronically excited states from where they can react with different rates than from the ground state $[9,15,16,36]$. To determine the ground-state reaction rate, we repeated the experiment by blocking the $397 \mathrm{~nm}$ laser during the reaction to only have $\mathrm{Ca}^{+}$ions in the electronic ground state. To stop the reaction, we closed the leak valve and unblocked the $397 \mathrm{~nm}$ laser again to recrystallize the Coulomb crystal prior to its ejection into the TOF spectrometer. From this experiment, we determined a second order rate constant for the reaction of $\mathrm{Ca}^{+}$with $\mathrm{N}_{2} \mathrm{O}$ of $5.49(32) \cdot 10^{-11} \mathrm{~cm}^{3} \mathrm{~s}^{-1}$ which agrees with the value reported by Spears and Fehsenfeld [33] but is a factor of 2.4 to 2.9 lower than the values reported by Plane et al. and Lavrov 
et al. [32, 34]. It should be emphasized, however, that because of the slight temperature dependence of this reaction and the non-thermal conditions prevailing in our experiment (the ions are not in thermal equilibrium because of their micromotion [6]), the comparison of our rate constant with the room-temperature values from the literature can only be approximate.

To test if we lose ions from the trap due to elastic collisions with $\mathrm{N}_{2} \mathrm{O}$, we blocked the $397 \mathrm{~nm}$ laser and leaked argon into the vacuum chamber for 90 seconds at the same partial pressures as in the $\mathrm{N}_{2} \mathrm{O}$ experiment. The crystal volume did not shrink more as in a control experiment in which no argon was admitted into the trap chamber. Therefore, we rule out any significant ion loss from elastic collisions.

We note that collisions with a buffer gas of higher mass than the trapped ions can lead to RF heating and loss of ions from the trap [37-39]. Therefore, the use of Ar which has almost the same mass as $\mathrm{Ca}$ as a non-reactive substitute to $\mathrm{N}_{2} \mathrm{O}$ has to be justified. However, the mass of $\mathrm{N}_{2} \mathrm{O}$ is only $4 \mathrm{u}$ larger than the one of $\mathrm{Ca}$ or $\mathrm{Ar}$ and the corresponding mass ratio $(m(\mathrm{Ar}) / m(\mathrm{Ca})=1.1)$ is below the critical ratio $\left(m_{c} \approx 1.24\right)$ at which significant ion heating is expected under our experimental conditions [40].

\section{Conclusions}

We designed and characterized a new linear quadrupole ion trap that is coupled to a timeof-flight mass spectrometer with a post-ejection-acceleration scheme that produces high resolution mass spectra with $m / \Delta m \approx 700$. The present setup was developed for studies of conformer-specific effects in ion-molecule reactions involving large organic ions and neutral molecules. The high resolution of our TOF-MS should enable us to unambiguously determine the mass of product ions in reactions of these complex species which is a key prerequisite for unraveling the underlying reaction mechanisms and dynamics.

Compared to previous reports of LQTs for cold ions coupled to a TOF mass spectrometer, we achieve a higher resolution at the cost of having to scan the post-ejectionacceleration delay over several experiments. In the TOF traps reported by Schowalter et al. and Deb et al. $[18,21]$, all ion masses can be assigned from a single experiment but these setups require more complicated RF electronics. By comparison, the present approach relies on simpler electronics at the expense of an increased mechanical complexity.

The post-ejection-acceleration delay does not have to be scanned over the full range to find the best setting. One can easily estimate the correct delay range from the low resolution TOF spectra and then scan over that range to find the optimal signal, as was done with the $\mathrm{CaOH}^{+}$ions in the present study. In this way high-resolution mass spectra for all ion species in the trap can be recorded in a reasonable amount of time.

By measuring the second order rate constant for the reaction: $\mathrm{Ca}^{+}+\mathrm{N}_{2} \mathrm{O} \rightarrow \mathrm{CaO}^{+}+$ $\mathrm{N}_{2}$, we have demonstrated that we can quantify the number of trapped ions. This makes our apparatus a versatile tool to investigate the rates of ion-molecule reactions.

\section{Acknowledgements}

We thank Prof. Jochen Küpper (Hamburg) for helpful discussions. We want to acknowledge the technical support from Dieter Wild and Georg Holderied. The present work is supported by the Swiss National Science Foundation under grant nr. BSCGI0_157874 and European Commission under the Seventh Framework Programme FP7 GA 607491 COMIQ. 


\section{Competing interests}

The authors declare that they have no competing interests.

Received: 15 April 2016 Accepted: 24 June 2016

Published online: 16 July 2016

\section{References}

1. Germann M, Tong X, Willitsch S (2014) Observation of electric-dipole-forbidden infrared transitions in cold molecular ions. Nat Phys 10:820-824

2. Blatt R, Wineland D (2008) Entangled states of trapped atomic ions. Nature 453:1008-1015

3. Tong X, Nagy T, Yosa Reyes J, Germann M, Meuwly M, Willitsch S (2012) State-selected ion-molecule reactions with Coulomb-crystallized molecular ions in traps. Chem Phys Lett 547:1-8

4. Hall FHJ, Aymar M, Bouloufa-Maafa N, Dulieu O, Willitsch S (2011) Light-assisted ion-neutral reactive processes in the cold regime: Radiative molecule formation versus charge exchange. Phys Rev Lett 107:243202

5. Hall FHJ, Aymar M, Raoult M, Dulieu O, Willitsch S (2013) Light-assisted cold chemical reactions of barium ions with rubidium atoms. Mol Phys 111:1683-1690

6. Willitsch S (2012) Coulomb-crystallised molecular ions in traps: methods, applications, prospects. Int Rev Phys Chem 31:175-199

7. Roth B, Blythe P, Wenz H, Daerr H, Schiller S (2006) lon-neutral chemical reactions between ultracold localized ions and neutral molecules with single-particle resolution. Phys Rev A 73:042712

8. Willitsch S, Bell MT, Gingell AD, Softley TP (2008) Chemical applications of laser- and sympathetically-cooled ions in ion traps. Phys Chem Chem Phys 10:7200-7210

9. Gingell AD, Bell MT, Oldham JM, Softley TP, Harvey JN (2010) Cold chemistry with electronically excited $\mathrm{Ca}^{+}$ Coulomb crystals. J Chem Phys 133:194302

10. Heazlewood BR, Softley TP (2015) Low-temperature kinetics and dynamics with Coulomb crystals. Annu Rev Phys Chem 66:475-495

11. Baba T, Waki I (2002) Spectral shape of in situ mass spectra of sympathetically cooled molecular ions. J Appl Phys 92:4109

12. Drewsen M, Mortensen A, Martinussen R, Staanum P, Sørensen JL (2004) Nondestructive identification of cold and extremely localized single molecular ions. Phys Rev Lett 93:243201

13. Staanum PF, Højbjerre K, Wester R, Drewsen M (2008) Probing isotope effects in chemical reactions using single ions. Phys Rev Lett 100:243003

14. Roth B, Blythe P, Schiller S (2007) Motional resonance coupling in cold multispecies Coulomb crystals. Phys Rev A 75:023402

15. Chang YP, Dlugolecki K, Küpper J, Rösch D, Wild D, Willitsch S (2013) Specific chemical reactivities of spatially separated 3-aminophenol conformers with cold Ca+ ions. Science 342:98-101

16. Rösch D, Willitsch S, Chang YP, Küpper J (2014) Chemical reactions of conformationally selected 3-aminophenol molecules in a beam with Coulomb-crystallized Ca+ ions. J Chem Phys 140:124202

17. Willitsch S, Bell MT, Gingell AD, Procter SR, Softley TP (2008) Cold reactive collisions between laser-cooled ions and velocity-selected neutral molecules. Phys Rev Lett 100:043203

18. Schowalter SJ, Chen K, Rellergert WG, Sullivan ST, Hudson ER (2012) An integrated ion trap and time-of-flight mass spectrometer for chemical and photo- reaction dynamics studies. Rev Sci Instrum 83:043103

19. Schneider C, Schowalter SJ, Chen K, Sullivan ST, Hudson ER (2014) Laser-cooling-assisted mass spectrometry. Phys Rev Appl 2:034013

20. Schneider C, Schowalter SJ, Yu P, Hudson ER (2016) Electronics of an ion trap with integrated time-of-flight mass spectrometer. Int J Mass Spectrom 394:1-8

21. Deb N, Pollum LL, Smith AD, Keller M, Rennick CJ, Heazlewood BR, Softley TP (2015) Coulomb crystal mass spectrometry in a digital ion trap. Phys Rev A 91:033408

22. Meyer KAE, Pollum LL, Petralia LS, Tauschinsky A, Rennick CJ, Softley TP, Heazlewood BR (2015) Ejection of Coulomb crystals from a linear Paul ion trap for ion-molecule reaction studies. J Phys Chem A 119:12449-12456

23. Ni KK, Loh H, Grau M, Cossel KC, Ye J, Cornell EA (2014) State-specific detection of trapped HfF ${ }^{+}$by photodissociation. J Mol Spectrosc 300:12-15

24. Jyothi S, Ray T, Rangwala SA (2015) Phase-sensitive radial extraction and mass spectrometry of trapped ions in a compact geometry. Appl Phys B 118:131-138

25. Seck CM, Hohenstein EG, Lien CY, Stollenwerk PR, Odom BC (2014) Rotational state analysis of AlH ${ }^{+}$by two-photon dissociation. J Mol Spectrosc 300:108-111

26. Franzen J (1998) Method and device for orthogonal ion injection into a time-of-flight mass spectrometer. U.S. patent. 5763878

27. Manura DJ, Dahl DA (2011) SIMION 8.0/8.1 User Manual, Rev. 5 edn. Scientific Instrument Services, Inc.. Ringoes, NJ

28. Matthey T, Cickovski T, Hampton S, Ko A, Ma Q, Nyerges M, Raeder T, Slabach T, Izaguirre JA (2004) Protomol, an object-oriented framework for prototyping novel algorithms for molecular dynamics. ACM Trans Math Softw 30:237-265

29. Mokhberi A, Willitsch S (2015) Structural and energetic properties of molecular Coulomb crystals in a surface-electrode ion trap. New J Phys 17:045008

30. March RE, Todd JF (2005) Quadrupole Ion Trap Mass Spectrometry. 2nd edn. John Wiley \& Sons, Hoboken

31. Drewsen M, Brøner A (2000) Harmonic linear Paul trap: Stability diagram and effective potentials. Phys Rev A 62:045401

32. Plane JMC, Vondrak T, Broadley S, Cosic B, Ermoline A, Fontijn A (2006) Kinetic study of the reaction $\mathrm{Ca}^{+}+\mathrm{N}_{2} \mathrm{O}$ from 188 to 1207 K. J Phys Chem A 110:7874-7881

33. Spears KG, Fehsenfeld FC (1972) Termolecular association reactions of Mg, Ca, and Ba ions. J Chem Phys 56:5698 
34. Lavrov W, Blagojevic V, Koyanagi GK, Orlova G, Bohme DK (2004) Gas-phase oxidation and nitration of first-, second-, and third-row atomic cations in reactions with nitrous oxide: Periodicities in reactivity. J Phys Chem A 108:5610-5624

35. Wiley WC, McLaren IH (1955) Time-of-flight mass spectrometer with improved resolution. Rev Sci Instrum 26:1150-1157

36. Okada K, Wada M, Boesten L, Nakamura T, Katayama I, Ohtani S (2003) Acceleration of the chemical reaction of trapped $\mathrm{Ca}^{+}$ions with $\mathrm{H}_{2} \mathrm{O}$ molecules by laser excitation. J Phys B: At Mol Opt Phys 36:33-46

37. Major FG, Dehmelt HG (1968) Exchange-Collision Technique for the rf Spectroscopy of Stored lons. Phys Rev 170:91

38. DeVoe RG (2009) Power-Law Distributions for a Trapped Ion Interacting with a Classical Buffer Gas. Phys Rev Lett 102:063001

39. Rouse I, Willitsch S (2015) Superstatistical velocity distributions of cold trapped ions in molecular-dynamics simulations. Phys Rev A 92:053420

40. Chen K, Sullivan ST, Hudson ER (2014) Neutral Gas Sympathetic Cooling of an Ion in a Paul Trap. Phys Rev Lett 112:143009

Submit your manuscript to a SpringerOpen ${ }^{\odot}$ journal and benefit from:

- Convenient online submission

- Rigorous peer review

- Immediate publication on acceptance

- Open access: articles freely available online

- High visibility within the field

- Retaining the copyright to your article

Submit your next manuscript at $>$ springeropen.com 